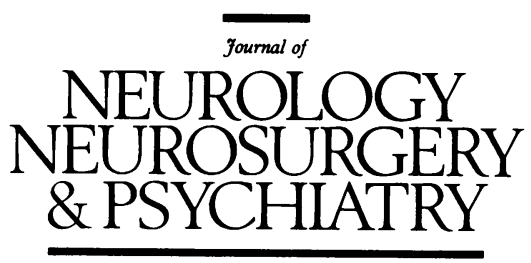

\title{
Ticlopidine, a new antithrombotic drug: but is it better than aspirin for longterm use?
}

Sound clinical trials of treatments to prevent stroke and other vascular events are time consuming, but if of sufficient size to give valid results can have a major impact on clinical practice. A critical review of the results of all randomised trials of antiplatelet agents available in $1988^{1}$ showed that for transient ischaemic attack (TIA) patients and the survivors of mild ischaemic strokes, $300 \mathrm{mg}$ of aspirin a day for a few years reduces the risk of serious vascular events (that is, stroke, myocardial infarction and vascular death) by about a quarter. Recently, clinically useful information is emerging from randomised clinical trials of ticlopidine, a new antiplatelet drug. Unlike aspirin, ticlopidine does not inhibit cyclo-oxygenase and, unlike dipyridamole, it does not inhibit phosphodiesterase. Nonetheless, it blocks the platelet release reaction, inhibits platelet aggregation induced by ADP and platelet adhesion, and prolongs the bleeding time.

The questions to be addressed are firstly, does ticlopidine have a clinically useful effect as an antithrombotic drug and secondly, if it does, how does it compare with aspirin?

\section{How effective is ticlopidine?}

The Canadian American Ticlopidine Study (CATS) ${ }^{2}$ randomised 1053 survivors of ischaemic stroke (about $70 \%$ of whom required some assistance in their activities of daily living) to ticlopidine $250 \mathrm{mg}$ bd versus placebo $1-17$ weeks post-stroke. Patients were followed for up to three years, the average being 24 months. As one would expect from the McMaster group, the trial was meticulously conducted and the sponsoring pharmaceutical company played no part in the data analysis. However, the authors concentrated their analysis not on all the eligible and randomised patients but on those whose outcome events occurred while taking trial medication or within 28 days of permanently and definitely stopping it. They also excluded four patients who had an intracranial haemorrhage during follow up (two in each treatment group). This so-called "efficacy" analysis usually provides the most optimistic estimate of a treatment effect since patients having an outcome event but not taking trial medication are excluded. However, sometimes such an analysis can be biased since patients may stop their trial medication as a result of a minor event (for example, a series of TIAs) which was, in fact, leading up to an important event (such as a stroke) that should have been included but, would not be if it occurs more than 28 days after treatment has been stopped.
It can also be very difficult to know precisely when patients stop trial medication, particularly if they die suddenly. This is, however, less of a problem if the analysis is done blind to treatment allocation, which it was in the CATS study.

However, the efficacy analysis in CATS demonstrated a $30.2 \%$ reduction $(95 \%$ confidence interval $7.5 \%-48.3 \%$ reduction) in the relative risk of the composite "end-point" of stroke/myocardial infarction/vascular death attributable to ticlopidine. Using such a composite end-point is justified since an antithrombotic drug is likely to influence favourably all three components, unlikely to influence nonvascular death which in fact was the case, and, by providing a larger number of events than its individual constitutents, increases the power of the analysis. The risk reduction for fatal and non-fatal recurrent stroke was similar at $33.5 \%$.

If all events in all eligible patients were counted, the relative risk reduction for stroke/myocardial infarction/ vascular death dropped to $23.3 \%$, and for fatal and nonfatal stroke to $20.5 \%$. The former was only just statistically significant and the $95 \%$ confidence interval was wide ( 1.0 to $40.5 \%$ risk reduction). This analysis was based on the more rigorous "intention-to-treat" principle in which every event in every randomised patient is counted up to the scheduled end of the trial whether the patient is taking trial medication or not. This is much preferred because, although less sensitive to a treatment effect, it is potentially less biased than an efficacy analysis.

Thus, it seems that ticlopidine almost certainly does reduce the risk of serious vascular events if taken for a year or two after a major ischaemic stroke but it is not certain how big that effect is; it could be less than a $10 \%$ relative risk reduction or it could be almost a $50 \%$ risk reduction. But what about the side effects? Clearly for some people ticlopidine is a mildly unpleasant drug to take since $12 \%$ of the patients stopped taking it compared with 3\% stopping placebo. The most common side effects were diarrhoea and skin rash but the most worrying was the occasional patient with neutropenia which implies that in routine clinical practice blood monitoring will be necessary for a few months after starting ticlopidine. This will certainly increase the cost of the treatment both directly and indirectly.

There is corroborative evidence supporting the efficacy of ticlopidine. In patients with intermittent claudication, a meta-analysis of trials of ticlopidine versus placebo has already suggested a therapeutic effect of ticlopidine on 


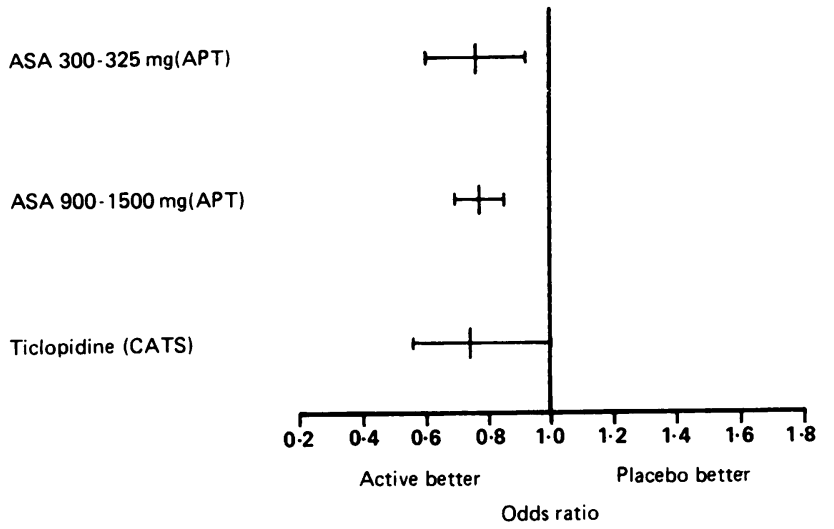

Figure 1 Indirect comparisons of different antiplatelet agents versus placebo in the longterm prevention of stroke/myocardial infarction/ vascular death in patients presenting with acute cerebrovascular and cardiovascular events. The results for aspirin come from the Antiplatelet Trialists' Collaboration (APT)' overview and for ticlopidine from the $C A T S$ trial. ${ }^{2}$ For each drug the result is expressed as an odds ratio (central vertical bar) and $95^{\circ}{ }_{\circ}$ confidence interval (horizontal lines).

vascular events, ${ }^{3}$ but so far only using data available from publications without additional data from the authors. More trials in various categories of vascular disease are in progress and as part of the Antiplatelet Trialists' Collaboration ${ }^{1}$ (APT) an extensive overview is in progress. However, evidence from the APT ${ }^{1}$ suggests after TIAs and mild ischaemic strokes aspirin $300 \mathrm{mg}$ daily reduces the risk of serious vascular events. This raises a further question.

\section{Is ticlopidine more or less effective than aspirin?}

This question can be addressed indirectly, but better directly. The indirect way is to compare the results of all the randomised trials of ticlopidine versus placebo (in fact this can only be done with the CATS trial which is the biggest, best, and most precise so far) with the results of all the randomised trials of aspirin versus placebo. The APT has provided an interim overview of antiplatelet drug efficacy and $300 \mathrm{mg}$ daily of aspirin is no less effective than higher doses. The efficacy of sulphinpyrazone was similar to aspirin alone, and aspirin combined with dipyridamole. Aspirin $300 \mathrm{mg}$ daily reduces the relative risk of stroke/ myocardial infarction/vascular death by $24 \%$ (95\% confidence interval $8 \%-40 \%$ reduction). This analysis is conservative since it is based on the "intention-to-treat" principle and should therefore be compared (indirectly) with a similar analysis of the CATS trial where the odds reduction (which is very similar to the risk reduction) was almost identical; $26 \%$ with a $95 \%$ confidence interval of 0 $45 \%$ (fig 1). This suggests therefore that there is little, if any, difference in efficacy between the two drugs but with a fairly wide margin of uncertainty because not only is the comparison indirect, but the confidence intervals for each estimate are fairly wide.

Table Ticlopidine Aspirin Stroke Study (TASS)

\begin{tabular}{|c|c|c|c|}
\hline Event & $\begin{array}{l}\text { Ticlopidine } \\
(n=1529) \\
250 \mathrm{mg} b d\end{array}$ & $\begin{array}{l}\text { Aspirin } \\
(n=1540) \\
650 \mathrm{mg} \text { bd }\end{array}$ & $\begin{array}{l}\text { Odds ratio } \\
\text { ( } 95 \% \text { confidence } \\
\text { interval) }\end{array}$ \\
\hline $\begin{array}{l}\text { Stroke and/or death } \\
\text { Stroke } \\
\text { Vascular death }\end{array}$ & $\begin{array}{l}306(19 \cdot 9) \\
172(11 \cdot 2)\end{array}$ & $\begin{array}{l}349(22 \cdot 8) \\
212(13 \cdot 9)\end{array}$ & $\begin{array}{l}0.85(0.72-1.02) \\
0.79(0.64-0.99)\end{array}$ \\
\hline $\begin{array}{l}\text { (inc. not known) } \\
\text { Non-vascular death } \\
\text { Death }\end{array}$ & $\begin{array}{rr}131 & (8 \cdot 6) \\
44 & (2 \cdot 9) \\
175 & (11 \cdot 4)\end{array}$ & $\begin{array}{rr}129 & (8 \cdot 4) \\
67 & (4 \cdot 4) \\
196 & (12 \cdot 7)\end{array}$ & $\begin{array}{l}1.02(0.70-1.51) \\
0.65(0.44-0.97) \\
0.89(0.70-1.11)\end{array}$ \\
\hline $\begin{array}{l}\text { Stroke/vascular } \\
\text { death`}\end{array}$ & $262(17 \cdot 1)$ & $282(18 \cdot 3)$ & $0.92(0.76-1.12)$ \\
\hline
\end{tabular}

*Approximate from published data because possibility of double counting ()$=\%$.

Intention-to-treat analysis based on calculation of odds ratios from $2 \times 2$ tables.

From Hass et al 1989.4

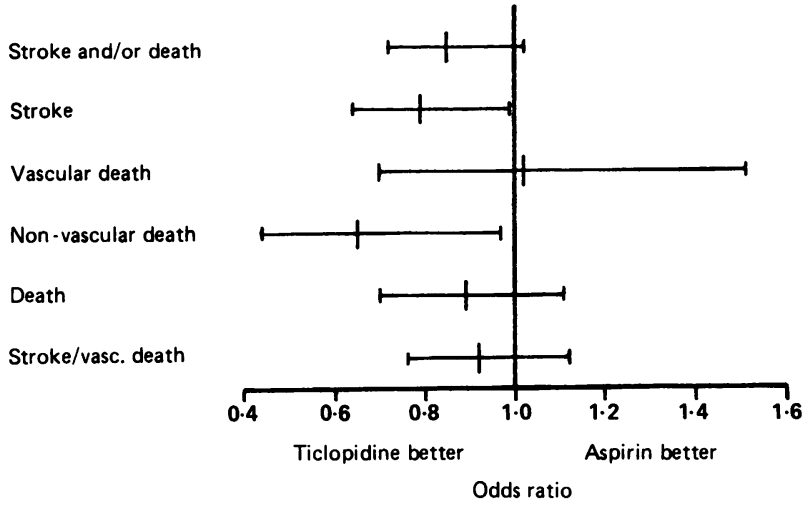

Figure 2 A representation of the TASS trial for various categories of event using the "intention-to-treat" analysis. For each category, the comparison of ticlopidine versus aspirin is displayed as an odds ratio (central vertical bar) and $95 \%$ confidence internal (horizontal lines).

However, we can compare the two drugs directly by looking at the recently published and competently performed Ticlopidine Aspirin Stroke Study (TASS) ${ }^{4}$ in which 3069 patients with a TIA or mild ischaemic stroke were randomised to ticlopidine $250 \mathrm{mg}$ bd versus aspirin $650 \mathrm{mg}$ bd. In the table, and fig 2 , I have reworked the published data as odds ratios using the "intention-to-treat" results provided by the authors. Unfortunately, non-fatal myocardial infarction was not recorded so it is probably best to concentrate on stroke, or stroke and/or vascular death. Taking the former there was a $21 \%$ relative reduction in the odds $(95 \%$ confidence interval $1 \%$ to $36 \%$ reduction), while in the latter the reduction was only $8 \%$ (95\% confidence interval $24 \%$ reduction to $12 \%$ increase) in favour of ticlopidine. Perusal of these results suggests that ticlopidine might be marginally more effective than aspirin but given the wide confidence intervals one cannot be absolutely sure.

Turning to side effects, it was quite clear that adverse effects were considerably more common in the ticlopidine group, particularly those causing premature termination of trial medication $(20.9 \%$ of patients on ticlopidine versus $14.5 \%$ of patients on what would now in the United Kingdom, at least, be regarded as rather an unnecessarily high dose of aspirin, $1300 \mathrm{mg}$ a day). Once again neutropenia was found to occur in a few cases and was severe enough to make it necessary for frequent blood monitoring in patients taking ticlopidine in routine clinical practice. Strangely, the most significant difference between ticlopidine and aspirin was in non-vascular death in favour of the former drug, presumably a chance effect because it was unexpected and applied as much to noncancer as cancer deaths. This also explains why the authors' preferred analysis based on stroke and/or death gave more promising results than my own based on stroke and/or vascular death which, if ticlopidine really has no effect on non-vascular death, is a more relevant "end-point" for assessing an antithrombotic drug.

It seems therefore that ticlopidine is not definitely more effective than aspirin in preventing serious vascular events in TIA and mild ischaemic stroke patients, and it is more toxic and expensive. Thus, there is no logical reason to start treatment with ticlopidine in vascular at-risk patients (that is, after TIA and ischaemic strokes). This raises a further question.

Which longterm antithrombotic treatment should be recommended if $300 \mathrm{mg}$ aspirin daily cannot be used because of previous or current gastric intolerance?

All the above arguments do presuppose that the effective- 
ness of long term antiplatelet agents is more or less the same after transient ischaemic attacks or mild ischaemic strokes (as addressed by the APT collaboration and TASS trial) as after more major ischaemic strokes (as addressed by the CATS trial). In other words, that it is biologically sensible, and therefore legitimate, to compare the effect of aspirin in TIA or mild ischaemic stroke patients with the effect of ticlopidine in major ischaemic stroke patients. Unfortunately, there have been no large trials of aspirin alone in major ischaemic stroke, and none at all of the direct comparison of aspirin against ticlopidine. Since it is unlikely that randomised comparisons of aspirin with ticlopidine in major ischaemic stroke patients will ever be done, we will have to make do with inferences from the data we do have.

It seems to me inherently unlikely that there is any qualitative difference between TIA and mild ischaemic stroke patients (indeed there is now evidence that this is true) $)^{5}$, nor any inherent reason why these two groups should be qualitatively any different from severe ischaemic stroke. There is similar underlying arterial pathology although the clinical effects of atheroma, thrombosis, and embolism are quantitatively different, hence the different clinical presentations of TIA and stroke. Also, as far as one can tell, the subsequent risk of serious vascular events is not very different after transient ischaemic attacks, mild ischaemic strokes, and more major ischaemic strokes; any differences there are between patients are probably more likely to be due to prognostic variables such as age, blood pressure, extent of arterial disease. Therefore, it is rather implausible that the relative risk reduction attributable to antiplatelet agents is going to be any different whether the patient presents with a TIA or a mild or major ischaemic stroke.

The alternative argument is to refuse to generalise from the APT and TASS trial, and conclude that aspirin should only be used for patients who recover from a cerebrovascular event, while ticlopidine should only be used in those who do not recover on the basis of CATS. However, it seems so implausible that the mere fact of recovery determines a clinically important difference between two antiplatelet drugs, that I prefer to generalise the results from the "TIA" trials to my individual stroke patients as well as TIA patients, and from the CATS trial in stroke to my individual "TIA" as well as stroke patients.

Therefore, if a TIA or ischaemic stroke patient of any grade of severity cannot take $300 \mathrm{mg}$ aspirin daily one might conclude from the TASS trial that he should take ticlopidine. But, it is worth reconsidering the APT results. ${ }^{1}$ This overview did suggest that sulphinpyrazone reduced the odds of stroke/myocardial infarction/vascular death by $17 \%$ (with a very wide $95 \%$ confidence interval from 1$33 \%$ reduction) but it was not conclusively more (or less) effective than aspirin alone. However, unlike ticlopidine, at least sulphinpyrazone is currently available in the United Kingdom, thus my recommendation would be that if aspirin cannot be used (even enteric coated in as low a dose as $300 \mathrm{mg}$ daily which is the lowest which definitely works in this situation) one can do one of two things; either use a lower dose still (150 mg or even $75 \mathrm{mg}$ daily) based on the pharmacological evidence that it should be as effective as the higher dose and backed up by some, but not many clinical trials, or, use sulphinpyrazone $200 \mathrm{mg}$ four times daily based on somewhat better clinical trial evidence but still short of complete certainty.

This conclusion will certainly need refining from the next cycle of the APT which should help clarify the place of ticlopidine in the longterm management of TIA and ischaemic stroke patients, and which will also direct priorities for future randomised clinical trials of ticlopidine.

Department of Clinical Neurosciences,

CHARLES WARLOW

Western General Hospital,

Edinburgh

1 Antiplatelet Trialists' Collaboration. Secondary prevention of vascular disease by prolonged antiplatelet treatment. Br Med $J$ 1988;296:320-31.

2 Gent M, Blakely JA, Easton JD, et al. The Canadian American Ticlopidine Study (CATS) in thromboembolic stroke. Lancet 1989;i:1215-20.

3 Boissel JP, Peyrieux JC, Destors JM. Is it possible to reduce the risk of cardiovascular events in subjects suffering from intermittent claudication of the lower limbs? Thromb Haemostasis 1989;62:1-3.

4 Hass WK, Easton JD, Adams HP, et al. A randomised trial comparing Ticlopidine hydrochloride with Aspirin for the prevention of stroke in high-risk patients. New Eng J Med 1989;321:501-7.

5 Dennis MS, Bamford JM, Sandercock PAGS, Warlow CP. A comparison of risk factors and prognosis for transient ischaemic attack and minor stroke. The Oxfordshire Community Stroke Project. Stroke 1989;20:1494-9. 